\title{
Efeito da dominância de membros sobre o desempenho em testes funcionais - Um estudo piloto
}

\section{Effect of limb dominance on functional tests performance - A pilot study}

\author{
Ana Isabela Teruyu ${ }^{1, *}$ \\ Caio de Paula Martins ${ }^{2}$ \\ Carla Paola Ferreira Felipe ${ }^{2}$ \\ Juliana Moraes Santos ${ }^{2}$ \\ Mayara Yamamoto ${ }^{2}$ \\ Rafael Mian da Silva ${ }^{2}$ \\ Rawane Karyn Oliveira Cardozo² \\ Cristiane Soncino Silva ${ }^{2}$ \\ Daniel Ferreira Moreira Lobato ${ }^{1}$
}

\section{Resumo}

Objetivo: Investigar a influência da dominância de membros sobre o desempenho dos membros inferiores em testes funcionais. Métodos: Oito atletas recreacionais $(20,70 \pm 2,19$ anos; $75,90 \pm 15,30 \mathrm{~kg}$; $1,73 \pm 0,07 \mathrm{~m})$, de ambos os sexos, fizeram parte deste estudo-piloto. O desempenho funcional dos membros inferiores foi avaliado por meio de três testes de salto unipodal: hop test (HP), six-meter timed hop test (STHT) e square hop test (SHT), sendo considerado, em cada teste, o desempenho médio de três tentativas válidas, para a análise dos dados. Resultados: De acordo com o teste t-Student para amostras dependentes $(\alpha=5 \%)$, não houve diferença significativa entre o desempenho dos membros dominante e não-dominante, nos três testes funcionais: HT $(p=0,27)$, STHT $(p=0,70)$ e SHT $(p=0,23)$. Conclusão: A dominância de membros inferiores parece não influenciar de forma decisiva 0 desempenho funcional de atletas recreacionais.

Palavras-Chave: dominância cerebral, membros inferiores, avaliação do desempenho.

\footnotetext{
Abstract

Objective: To investigate the influence of limb dominance on lower limb performance in functional tests. Methods: Eight recreational athletes $(20.70 \pm 2.19$ years, $75.90 \pm 15.30 \mathrm{~kg}, 1.73 \pm 0.07 \mathrm{~m})$, of both sexes, were took part of this pilot-study. The lower limb functional performance was evaluated by means of three single-leg hop tests: hop test (HP), six-meter timed hop test (STHT) and square hop test (SHT). Each test considered the average performance of three valid attempts to data analyzes. Results: According to the Student's t-test for dependent samples ( $\alpha=5 \%)$, there was no significant difference between dominant and non-dominant limbs performance in the three functional tests: HT $(p=0.27)$, STHT $(p=0.70)$ and SHT $(p=0.23)$. Conclusion: The lower limb dominance does not seem to influence decisively the functional performance of recreational athletes.
}

Keywords: cerebral dominance, lower extremity, performance tests.

\section{Afiliação dos autores}

${ }^{1}$ Universidade Federal do Triângulo Mineiro, Uberaba, Minas Gerais,

Brasil.

${ }^{2}$ Centro Universitário UNISEB,

Ribeirão Preto, São Paulo, Brasil.

${ }^{\star}$ Autor correspondente

Departamento de Fisioterapia Aplicada, Rua Vigário Carlos, 100, Bloco B, 4 andar, Sala 406, Abadia CEP: 38025-350, Uberaba, Minas Gerais, Brasil.

e-mail: anaiteruyu@gmail.com

Conflito de interesses

Os autores declararam não haver conflito de interesses.

Processo de arbitragem 


\section{Introdução}

A avaliação funcional do membro inferior após uma lesão é necessária para que o fisioterapeuta acompanhe e monitore efetivamente o processo de reabilitação ${ }^{1}$. Durante as avaliações de rotina clínica, os profissionais geralmente utilizam o desempenho do membro não lesionado como uma referência para essa comparação com o membro lesionado. Contudo, questiona-se a viabilidade desse procedimento, em virtude dos possíveis efeitos relacionados à dominância de membros ${ }^{2}$.

O conceito de dominância ainda não é bem definido, mas de maneira geral é determinado através das características de preferência na utilização de um membro para a execução de tarefas que exigem força, equilíbrio, coordenação e propriocepção $0^{3,4}$. Uma possível explicação para a assimetria motora entre os membros é consequência da lateralidade humana, representada por uma diferença na atividade entre os dois hemisférios cerebrais, manifestando-se de forma desigual para os membros em diferentes atividades ${ }^{3,5}$.

Sugere-se que as diferenças bilaterais entre membros podem representar uma forma de assimetria funcional, definida como uma discrepância consistente no uso dos membros dominante e não-dominante na realização de tarefas ${ }^{6}$. Deste modo, podem afetar a habilidade individual durante a realização de tarefas unilaterais e bilaterais envolvendo descarga de peso corporal $^{7}$, o que pode aumentar o risco de lesões (e recidivas) no membro inferior ${ }^{7,8}$. Neste sentido, a falha em considerar a existência de diferenças bilaterais relacionadas à dominância ou à lateralidade pode ser problemática em situacões de teste em que um membro serve de controle para o outro ${ }^{\text {. }}$.

Contudo, os efeitos da dominância de membros sobre o desempenho funcional ainda não são muito claros. A maioria dos estudos consultados não verificou diferenças entre os membros dominante e não-dominante quanto à força muscular ${ }^{1,8-15}$ e quanto ao desempenho em testes funcionais ${ }^{9,13,14}$. Controversamente, estudos epidemiológicos têm evidenciado que não apenas os desequilíbrios de força existem, mas que eles podem resultar em um aumento no risco de lesões para atletas que apresentem diferenças bilaterais superiores a $10 \%{ }^{16}$.

Uma provável razão para a inconsistência entre os resultados dos estudos experimentais e epidemiológicos ocorre em função do fato de que a maioria desses estudos ${ }^{12,14,17,18}$ avaliou 0 desempenho dos membros inferiores apenas considerando a função muscular concêntrica ou isométrica, diferentemente de outros estudos ${ }^{1,9,15,19}$ que avaliaram a força concêntrica e excêntrica. Sabe-se que a maioria das atividades esportivas apresenta uma grande demanda de ação muscular excêntrica, caracterizada por forças de aceleração e desaceleração de alta intensidade, e que esta ação está diretamente relacionada ao controle de movimentos articulares excessivos, que representam um dos principais fatores para as lesões esportivas ${ }^{20}$. Contudo, até o momento, menor atenção foi destinada ao estudo desse tipo de ação muscular.

Além disso, a comparação de déficits bilaterais no desempenho funcional pode apresentar um papel crucial para o fisioterapeuta, dada à aproximação desses testes com os gestuais esportivos. Saltos em distância ou que ocorrem em função do tempo são comumente utilizados para avaliar a função do joelho em pacientes com lesão do ligamento cruzado anterior (LCA) e designados a refletir a demanda de uma atividade física de alto nível ${ }^{8,13,21}$. Portanto, supõe-se que pesquisas sobre esse assunto fornecerão melhor suporte às avaliações prévias à participação de um atleta em atividades de alta intensidade, ou ainda em períodos de pré-temporada, com o objetivo de detectar assimetrias funcionais, direcionar intervenções voltadas à correção desses déficits e, consequentemente, reduzir os riscos de lesão $0^{1,22}$.

Considera-se que uma correta compreensão dos efeitos da dominância de membros sobre o desempenho funcional permitirá ao clínico aprimorar as suas habilidades em avaliar as lesões do membro inferior, bem como a sua progressão, especialmente no âmbito esportivo. Deste modo, este estudo piloto teve por objetivo investigar a influência da dominância de membros sobre o desempenho dos membros inferiores em testes funcionais, em atletas recreacionais.

\section{Métodos}

\section{Participantes}

Oito atletas recreacionais $(20,75 \pm 2,19$ anos; $75,90 \pm 15,35 \mathrm{~kg}$ $1,73 \pm 0,07 \mathrm{~m}$ ), de ambos os sexos (sendo 5 homens e 3 mulheres), com idade entre 19 e 25 anos, participaram deste estudo piloto, de caráter experimental e transversal, realizado no ano de 2017. Um atleta recreacional foi considerado como aquele praticante de atividades físicas regulares com uma frequência mínima de 3 vezes por semana, no mínimo de 30 minutos por sessão ${ }^{23}$.

Foram considerados como critérios de não-inclusão: a) idade inferior a 18 anos e superior a 26 anos; b) condição de sedentarismo ou de prática de atividade física em nível inferior ao utilizado para classificar os atletas como recreacionais; c) presença de dores ou disfunções relacionadas aos complexos articulares do membro inferior; d) existência de cirurgias prévias no membro inferior e e) presença de disfunções cárdiorespiratórias que limitassem a realização da atividade. Todos os voluntários assinaram um Termo de Consentimento previamente à participação no estudo, sendo a pesquisa previamente aprovada pelo Comitê de Ética em Seres Humanos da Universidade Federal do Triângulo Mineiro (parecer $\mathrm{n}^{\circ}$ 2.073.109/2017).

\section{Procedimentos}

Os voluntários foram submetidos a uma avaliação inicial para a coleta de dados clínicos e físicos, bem como para a verificação dos critérios de inclusão e não-inclusão no estudo. A dominância de membros foi determinada perguntando aos atletas qual o membro que eles utilizavam para chutar uma bola na máxima distância possível ${ }^{15}$. $O$ membro inferior direito foi o dominante em seis atletas (75\%). Por fim, foi sorteado qual membro (dominante ou não-dominante) seria primeiramente submetido aos testes funcionais, bem como a ordem de avaliação dos testes, para cada voluntário.

O desempenho funcional foi avaliado por meio de três testes de ampla utilização clínica para a avaliação do membro inferior: hop test, six-meter timed hop test e square hop test. Os sujeitos receberam uma breve explicação sobre os testes funcionais e assistiram a uma demonstração de realização dos mesmos. Em seguida, foram submetidos a uma familiarização com cada teste, para garantir a adequada compreensão das ações requeridas e dos procedimentos envolvidos. Além disso, esse procedimento consistiu em uma medida de aquecimento específico para cada tarefa a ser realizada.

Para a realização do hop test $(\mathrm{HT})$, os atletas foram instruídos a permanecer em apoio simples imediatamente atrás de uma marcação inicial. Com os membros superiores livres ao lado do corpo, os atletas deveriam saltar a máxima distância horizontal possível, aterrissando unicamente sobre o membro inferior que realizou o salto (Figura 1), com a medida de distância saltada (em metros e centímetros) sendo registrada pelos examinadores por meio de uma fita métrica comum.

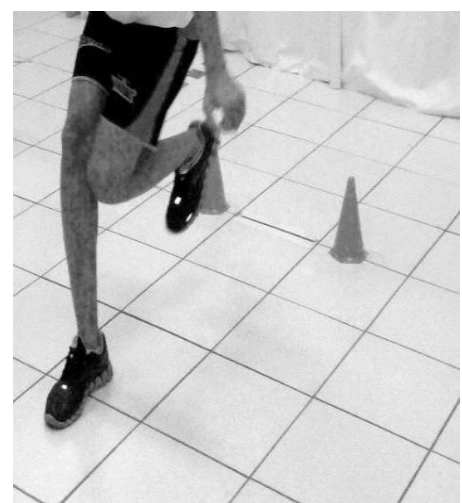

Figura 1. Realização do hop test com o membro inferior direito.

O teste foi executado em três tentativas válidas, sendo a média dos resultados obtidos utilizada para a análise dos dados. Os critérios para considerar uma tentativa válida foram a manutenção do equilíbrio em apoio unipodal, sobre o membro inferior que realizou o salto, por um período mínimo de 2 segundos, sem a perda de equilíbrio, sem tocar com a 
extremidade inferior contralateral ou com a extremidade superior no solo, ou ainda sem realizar um salto adicional durante a aterrissagem. Foi respeitado um intervalo de repouso de 30 segundos entre cada tentativa. $\mathrm{O}$ mesmo protocolo foi repetido para o membro contralateral.

Para a realização do six-meter timed hop test (STHT), os atletas foram instruídos a permanecer em apoio simples imediatamente atrás de uma marcação inicial. Com os membros superiores livres ao lado do corpo, os atletas deveriam percorrer uma distância de seis metros (demarcada no local do exame por cones) saltando na máxima velocidade possível e sem desacelerar ao longo do teste (Figura 2), com a medida de tempo decorrido (em segundos e centésimos de segundo) sendo registrada pelos examinadores por meio de um cronômetro digital.

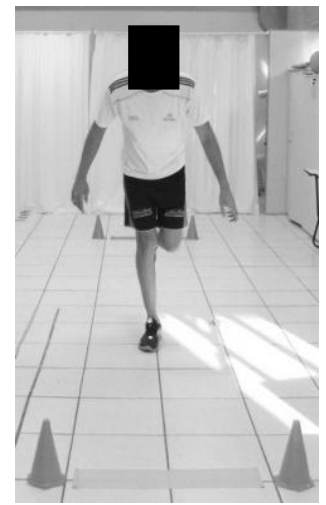

Figura 2. Realização do six-meter timed hop test com o membro inferior direito.

O teste foi executado em três tentativas válidas, sendo a média dos resultados obtidos utilizada para a análise dos dados. Os critérios para considerar uma tentativa válida foram a manutenção do equilíbrio ao longo da atividade, sobre o membro inferior que realizou os saltos, sem tocar com a extremidade inferior contralateral ou com a extremidade superior no solo, bem como a manutenção de um estado de aceleração máxima ao longo do teste. Foi respeitado um intervalo de repouso de 60 segundos entre cada tentativa. $\mathrm{O}$ mesmo protocolo foi repetido para o membro contralateral.

Para a realização do square hop test (SHT), os atletas foram instruídos a permanecer em apoio simples imediatamente atrás de uma marcação em forma de quadrado (44 cm X $44 \mathrm{~cm})$. Com os membros superiores livres ao lado do corpo, os atletas deveriam saltar para dentro e para fora desse quadrado (em sentido anti-horário para o membro inferior direito e em sentido horário para o membro inferior esquerdo), da forma mais rápida e precisa possível (Figura 3), durante o período de 30 segundos, registrados por cronômetro. O examinador observava a realização do teste e considerava o número de saltos realizados com aterrissagem do pé inteiramente dentro da marcação em quadrado.

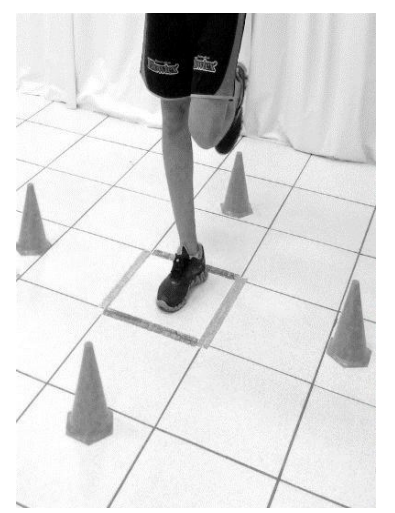

Figura 3. Realização do square hop test com o membro inferior direito.

O teste foi executado em três tentativas válidas, sendo a média dos resultados obtidos utilizada para a análise dos dados.
Os critérios para considerar uma tentativa válida foram a manutenção do equilíbrio ao longo da atividade, sobre o membro inferior em avaliação, sem tocar com a extremidade inferior contralateral ou com a extremidade superior no solo. Foi respeitado um intervalo de repouso de 60 segundos entre cada tentativa. O mesmo protocolo foi repetido para o membro contralateral.

\section{Análise dos dados}

Os dados de desempenho funcional foram analisados por meio do software STATISTICA 5.0 for Windows (StatSoft, Inc, Tulsa, USA). O teste de Shapiro Wilk foi utilizado para testar a normalidade dos conjuntos de dados e, uma vez que tais critérios foram satisfeitos, utilizou-se o teste $t$-Student para amostras dependentes para a comparação do desempenho funcional entre os membros dominante e não-dominante, considerando um nível de significância de 5\%

\section{Resultados}

Não houve diferença significativa entre o desempenho funcional dos membros dominante e não-dominante em qualquer um dos testes funcionais realizados: $H T(p=0,27)$, STHT $(p=0,70)$ e SHT $(p=0,23)$, embora tenha sido observado um melhor desempenho para o membro dominante no HT e do membro não-dominante no STHT e no SHT (Tabela 1).

Tabela 1

Média e desvio-padrão do desempenho funcional dos membros dominante e não-dominante - $(n=08)$.

\begin{tabular}{ccccc}
\hline & MDOM & MNDOM & $\begin{array}{c}\text { Diferença } \\
(\%)\end{array}$ & $\begin{array}{c}p \text { - } \\
\text { valor }\end{array}$ \\
\hline HT $(m)$ & $1,78 \pm 0,48$ & $1,75 \pm 0,45$ & 1,71 & 0,27 \\
STHT (s) & $2,20 \pm 0,48$ & $2,15 \pm 0,39$ & 2,32 & 0,70 \\
SHT (saltos) & $16,96 \pm 7,80$ & $18,50 \pm 6,56$ & 9,08 & 0,23 \\
\hline
\end{tabular}

(

STHT: six-meter timed hop test; SHT: square hop test.

\section{Discussão}

Este estudo piloto surgiu da necessidade em comparar as diferenças no desempenho funcional entre os membros dominante e não-dominante em atletas. Nenhuma diferença significativa entre os membros foi encontrada, o que concorda direta $^{9,13,21,24-27}$ e indiretamente $e^{1,11-15}$ com diversos estudos, porém discorda indiretamente de outros ${ }^{22,28,29}$.

Alguns estudos investigaram o efeito da dominância de membros sobre o torque extensor do joelho, nas modalidades concêntrica $^{12,14,17,18}$ e concêntrica e excêntrica ${ }^{1,9,15,19}$ e não verificaram diferenças significativas entre a força dos membros dominante e não-dominante. Da mesma forma, não foram constatadas diferenças bilaterais na carga utilizada durante o exercício de agachamento unipodal, tanto em homens quanto em mulheres ${ }^{1}$. Em conjunto, esses estudos indicam similaridade de desempenho entre os membros dominante e não-dominante, tanto para atividades realizadas em cadeia cinética aberta quanto em cadeia cinética fechada.

Contudo, resultados contrários foram verificados em outros estudos, que identificaram maior força no membro dominante de adultos jovens ${ }^{22}$ e em jogadores de futebol ${ }^{29}$. Ainda, destacamse os achados de Kong e Burns ${ }^{28}$, que evidenciaram que o pico de torque isométrico e isocinético dos isquiotibiais a $300 \%$ foi maior no membro dominante do que no membro contralateral. Assim, esses estudos indicam melhor desempenho do membro dominante em relação ao não-dominante.

Apesar dos resultados controversos sobre o tema, destacase que a maioria dos estudos que investigaram o desempenho em atletas recreacionais (como o realizado no presente estudo) não encontraram diferenças bilaterais significativas ${ }^{9}$. Hipotetizase que, nestes casos, parece haver um ambiente menos favorável ao desenvolvimento de assimetrias funcionais, uma vez que o trabalho requerido para a maioria das atividades do dia a dia é usualmente bilateral e que o tempo de exposição à prática esportiva desses voluntários pode ser insuficiente para a instalação de diferenças significativas entre o desempenho dos membros dominante e não-dominante, ao contrário do que pode ocorrer em esportes que requeiram maior demanda funcional sobre um dos membros inferiores ${ }^{29}$. 
Observa-se ainda que, embora haja diferenças entre os estudos supracitados quanto aos músculos testados, a ação muscular estudada, a velocidade e o tipo de exercício utilizado nos testes, a forma de familiarização dos sujeitos, o tempo de repouso, as variáveis mensuradas e o perfil da amostra avaliada, considera-se que as comparações entre membros continuam sendo de importante indicação, uma vez que os métodos utilizados para avaliar um dos membros, em cada estudo, foram idênticos àqueles utilizados para avaliar o membro contralateral.

É importante mencionar que, apesar da relevância das medidas de força como uma forma de avaliar se e como a fraqueza muscular pode afetar o recrutamento de unidades motoras, uma avaliação funcional parece necessária para oferecer maiores detalhes sobre o desempenho muscular, uma vez que simula situações que são comuns às atividades esportivas. Neste sentido, os resultados do presente estudo concordam com outros trabalhos que utilizaram o single leg hop test $^{13,21,24,25}$, o vertical jump ${ }^{13,24,21,26}$, o single leg hop for time ${ }^{24}$, o side hop test $t^{27}$, o 6-meter crossover hop test ${ }^{27}$, o square hop test $t^{27}$, o figure-of-8 test $t^{27}$, o shuttle run ${ }^{21}$ e, portanto, não verificaram diferenças significativas entre os membros dominante e não-dominante nos testes funcionais citados.

Deste modo, os resultados do presente estudo sugerem a existência de uma simetria funcional entre os membros inferiores em atletas recreacionais, e que a dominância de membros parece não ter efeito sobre essa condição. Esses resultados são úteis na medida em que fornecem informações adicionais sobre o desempenho funcional de atletas, considerando os aspectos relacionados à dominância de membros e, ao mesmo tempo, são necessários ao conhecimento do fisioterapeuta devido ao amplo uso desses testes no ambiente clínico, tanto para avaliação, quanto para a reabilitação de atletas.

Clinicamente, outro aspecto que parece relevante diz respeito à possibilidade de eliminação de escores pré-teste como medida de linha de base ou o uso de um grupo controle externo $^{30}$, uma vez que o membro não lesionado poderia ser utilizado para comparação, independente dos efeitos de dominância, desde que os indivíduos não realizem atividade assimétrica repetitiva. Após uma lesão, os sujeitos tendem a mudar a sua preferência de membros inferiores, com ou sem uma mudança funcional de dominância associada. Esta é outra relação em que as informações a respeito das adaptações fisiológicas frente a uma lesão podem ser benéficas para o clínico $^{2}$. Quando uma grande assimetria estiver presente, ela pode ocorrer em função de uma lesão aguda ou crônica e não devido à dominância de membros.

Este estudo apresenta algumas limitações. Primeiramente, a amostra utilizada foi de conveniência. Como consequência, ela pode ser mais saudável do que a população em geral e é menos condicionada do que uma população de atletas de elite. Ainda, a composição da amostra envolve homens e mulheres, portanto, heterogênea em relação ao sexo. Desta forma, esses resultados devem ser analisados com cautela, e não devem ser aplicados a pacientes ou atletas de elite, bem como considerar exclusivamente um sexo. Além disso, em virtude do reduzido tamanho amostral, esses resultados não podem ser generalizados dentro da população de atletas recreacionais. Entretanto, ressaltamos que trata-se de um estudo piloto, que deverá ser expandido para permitir resultados mais expressivos sobre o tema.

Por fim, uma consideração em especial deve ser realizada em relação ao método utilizado para classificar a dominância de membros. Os voluntários foram instruídos a indicar com qual perna eles preferencialmente chutariam uma bola, e o membro reportado para 0 chute foi considerado como o dominante ${ }^{15}$. Contudo, existe a possibilidade de que este membro não represente aquele mais utilizado em tarefas que não envolvam o chute, tais como o salto e a aterrissagem. Enquanto a assimetria funcional em membros superiores parece óbvia ${ }^{3}$, existem diversos testes para avaliar a dominância de membros inferiores. Estas tarefas incluem chutar uma bola, subir degraus de uma escada, e acompanhar com os pés o ritmo de uma melodia, por exemplo. Nestas atividades, o membro nãodominante é geralmente utilizado como suporte de peso corporal, enquanto o membro dominante provê a propulsão para realizar tarefas mais complexas ${ }^{6}$.

Uma vez que não há critério claro para validar as medidas adotadas no presente estudo, a seleção de procedimentos para classificar a dominância de membros deve ser guiada por predileção pessoal. Consequentemente, um estudo tem variado em relação ao outro na forma de avaliar a dominância de membros inferiores, tornando difícil determinar se as diferenças de resultados encontradas entre os estudos são significativas ou simplesmente consequência de uma variação metodológica. Deste modo, os resultados controversos entre os estudos consultados podem ser decorrentes de diferentes desenhos experimentais, incluindo o modo de determinação da dominância de membros.

Por fim, fatores de cunho psicológico, como o estado de motivação dos voluntários para a execução dos testes e o fenômeno da habituação com os mesmos, sempre devem ser considerados como relevantes ao se avaliar o desempenho muscular. Entretanto, a padronização do comando verbal, a utilização de apenas um examinador para conduzir cada avaliação, bem como a devida familiarização dos voluntários com todas as etapas do teste foram adotadas como estratégias experimentais para reduzir a possibilidade de interferência desses fatores.

\section{Conclusão}

A dominância de membros parece não alterar de forma significativa 0 desempenho funcional do membro inferior. Entretanto, destaca-se o seu caráter ainda preliminar que, associado a uma amostra de maior representatividade e ao estudo de outras variáveis do desempenho muscular, poderá levar a resultados mais conclusivos sobre este tema.

\section{Referências}

1. McCurdy K, Langford G. Comparison of unilateal squat strength between the dominant and non-dominant leg in men and women. J Sports Sci Med. 2005 (4), 153-159.

2. Hoffman M, Schrader J, Applegate T, Koceja D. Unilateral Postural Contro of the Functionally Dominant and Nondominant Extremities of Healthy Subjects. J Athl Train. 1998; 33(4):319-322.

3. Amaro EA, Raimundo KC, Santos NTO, Bonfim PBS, Walsh IAP, Bertoncello D. Universitários canhotos: dificuldades encontradas propostas para as atividades cotidianas. Cad Ter Ocup UFSCar. 2014; 22(3):531-6.

4. Gabbard C, Hart S. A Question of Foot Dominance. J Gen Psychol. 1996; 123(4):289-96.

5. Barut C, Ozer CM, Sevinc O, Gumus M, Yunten Z. Relationships between hand foot preferences. Int J Neurosci. 2007;117(2):177-85.

6. Seeley MK, Umberger BR, Shapiro R. A test of the functional asymmetry hypothesis in walking. Gait Posture. 2008; 28(1):24-8.

7. Hewett TE, Myer GD, Ford KR. Prevention of anterior cruciate ligament injuries. Curr Womens Health Rep. 2001; (1):218-24.

8. Paterno MV, Rauh MJ, Schmitt LC, Ford KR, Hewett TE. Incidence of contralateral and Ipsilateral Anterior Cruciate Ligament (ACL) Injury After Primary ACL Reconstruction and Return to Sport.Clin J Sport Med. 2012; 22(2):116-21.

9. Lobato DFM, Nakanishi V, Shibuya C, Serrão FV. Effects of Lower-Limb Dominance on Isokinetic and FunctionalProfiles in Healthy Young Active Men - A Pilot Study. EC Orthopaedics. 2018; 9(7):477-486.

10. Delextrat A, Baker J, Cohen DD, Clarke ND. Effect of a simulated soccer match on the functional hamstrings-to-quadriceps ratio in amateur female players: H: Q ratio in female soccer players. Scand J Med Sci Sports. 2013; 23(4):478-86.

11. Lobato DFM, Silva CS, Santos JM, Lopes LO. Efeito do alongamento muscular agudo sobre a força de preensão manual - Um estudo preliminar. Revista Multidisciplinar de Iniciação Científica do Centro Universitário UNISEB - COC. 2011; (2):172-181.

12. Masuda K, Kikuhara N, Takahashi H, Yamanaka K. The relationship between muscle cross-sectional area and strength in various isokinetic movements among soccer players. J Sports Sci. 2003; 21(10):851-8.

13. Petschnig $R$, Baron $R$, Albrecht $M$. The Relationship Between Isokinetic Quadriceps Strength Test and Hop Tests for Distance and One-Legged Vertical Jump Test Following Anterior Cruciate Ligament Reconstruction. J Orthop Sports Phys Ther. 1998; 28(1):23-31.

14. Greenberger HB, Paterno MV. Relationship of Knee Extensor Strength and Hopping Test Performance in the Assessment of Lower Extremity Function. J Orthop Sports Phys Ther. 1995; 22(5):202-6.

15. Hageman PA, Gillaspie DM, Hill LD. Effects of Speed and Limb Dominance on Eccentric and Concentric Isokinetic Testing of the Knee. J Orthop Sports Phys Ther. 1988; 10(2):59-65.

16. Knapik JJ, Bauman CL, Jones BH, HarrisJM, Vaughan L. Preseason strength and flexibility imbalances associated with athletic injuries in female collegiate athletes. Am J Sports Med. 1991; (19):76-81.

17. Almeida GPL, Carneiro KKA, Morais HCR, Oliveira JBB. Efeitos da dominância unilateral dos membros inferiores na flexibilidade e no desempenho isocinético em mulheres saudáveis. Fisioter. Mov. 2012; 25(3):551-559.

18. Hunter SK, Thompson MW, Adams RD. Relationships Among AgeAssociated Strength Changes and Physical Activity Level, Limb Dominance, and Muscle Group in Women. J Gerontol A Biol Sci Med Sci. 2000, 55(6):264-273. 
19. Miller LE, Pierson LM, Nickols-Richardson SM, Wootten DF, Selmon SE, Ramp WK, et al. Knee Extensor and Flexor Torque Development With Concentric and Eccentric Isokinetic Training. Res Q Exerc Sport. 2006; 77(1):58-63.

20. Franchi MV, Reeves ND, Narici MV. Skeletal Muscle Remodeling in Response to Eccentric vs. Concentric Loading: Morphological, Molecular, and Metabolic Adaptations. Front Physio. 2017; (8): 447.

21. Barber SD, Noyes FR, Mangine RE, Mccloskey JW, Hartman W. Quantitative Assessment of Functional Limitations in Normal and Anterior Cruciate Ligament-Deficient Knees. Clin Orthop Relat Res. 1990; (255):204-214

22. Ross S, Guskiewicz K, Prentice W, Schneider R, Yu B. Comparison of Biomechanical Factors between the Kicking and Stance Limbs. J Sport Rehabil. 2004; 13(2):135-50.

23. Heinert BL, Kernozek TW, Greany JF, Fater DC. Hip Abductor Weakness and Lower Extremity Kinematics during Running. J Sport Rehabil. 2008;17(3):243-56.

24. Swearingen J, Lawrence E, Stevens J, Jackson C, Waggy C, Davis DS. Correlation of single leg vertical jump, single leg hop for distance, and single leg hop for time. Phys Ther Sport. 2011; 12(4):194-8.
25. Kobayashi Y, Kubo J, Matsubayashi T, Matsuo A, Kobayashi K, Ishii N. Relationship between Bilateral Differences in Single-Leg Jumps and Asymmetry in Isokinetic Knee Strength. J Appl Biomech. 2013; 29(1):61-7.

26. Mcelveen MT, Riemann BL, Davies GJ. Bilateral comparacion of propulsion mechanics during single-leg vertival jumping. J Strength Cond propulsion mechanics during

27. Caffrey E, Docherty CL, Schrader J, Klossnner J. The Ability of 4 SingleLimb Hopping Tests to Detect Functional Performance Deficits in Individuals With Functional Ankle Instability. J Orthop Sports Phys Ther 2009; 39(11):799-806.

28. Kong PW, Burns SF. Bilateral difference in hamstrings to quadriceps ratio in healthy males and females. Phys Ther Sport. 2010; 11(1):12-7.

29. Kellis S, Gerodimos V, Kellis E, Manou V. Bilateral isokinetic concentric and eccentric strength profiles of the knee extensors and flexors in young soccer players. Isokinet Exerc Sci. 2001; (9):31-39.

30. Van Cingel REH, Gertjan Kleinrensink, Elian J. Uitterlinden, Patrick PGM Rooijens, Paul GH Mulder, Geert Aufdemkampe, et al. Repeated Ankle Sprains and Delayed Neuromuscular Response: Acceleration Time Parameters. J Orthop Sports Phys Ther. 2006; 36(2):72-79. 